\title{
ON THE LITTLEWOOD-PALEY THEORY FOR MIXED NORM SPACES
}

BY

\author{
JOHN A. GOSSELIN ${ }^{1}$
}

\begin{abstract}
An inequality of Littlewood-Paley type is proved for the mixed norm spaces $L_{p}\left(l_{r}\right), 1<p, r<\infty$, on the interval $[0,1]$. This result makes use of recent work by $C$. Fefferman and $A$. Cordoba on the boundedness of singular integrals on these spaces. As an application of this inequality, boundedness of the lacunary maximal partial sum operator for Walsh-Fourier series on $L_{p}\left(l_{r}\right)$ is established. This result can be viewed as an extension of a similar result for the Hardy-Littlewood maximal function due to $C$. Fefferman and E. M. Stein.
\end{abstract}

Introduction. Recently various authors (see [1], [3], [4], and [11], for example) have established the boundedness of certain (sub-)linear operators on mixed norm spaces. In this paper we prove similar results for a version of the Littlewood-Paley function and for the lacunary maximal partial sum operator for Walsh-Fourier series. A particular case of the latter result is the Fefferman-Stein inequality proved in [3]. A by-product of our proofs is an extension of Khinchine's inequality to mixed norm spaces.

The author wishes to thank N. R. Ladhawala, W. S. Young, and the referee for suggesting certain simplifications.

I. In this section we extend an inequality of Khinchine [12] to mixed norm spaces.

LEMMA 1. Let $\left\{f_{k}\right\}_{k=1}^{\infty}$ denote a sequence of functions on $[0,1]$, each being given by a Rademacher series $\sum_{v=1}^{\infty} a_{v}^{k} r_{p}(t)$. Let $1<p, r<\infty$. Then there exist constants $C_{p, r}$ and $B_{p, r}$ depending only on $p$ and $r$ such that

$$
\left(\int_{0}^{1}\left(\sum_{k=1}^{\infty}\left|f_{k}(x)\right|^{r}\right)^{p / r} d x\right)^{1 / p}<B_{p, r}\left(\sum_{k=1}^{\infty}\left(\sum_{\nu=1}^{\infty}\left|a_{\nu}^{k}\right|^{2}\right)^{r / 2}\right)^{1 / r}
$$

and

$$
\left(\int_{0}^{1}\left(\sum_{k=1}^{\infty}\left|f_{k}(x)\right|^{r}\right)^{p / r} d x\right)^{1 / p} \geqslant C_{p, r}\left(\sum_{k=1}^{\infty}\left(\sum_{\nu=1}^{\infty}\left|a_{\nu}^{k}\right|^{2}\right)^{r / 2}\right)^{1 / r} .
$$

Received by the editors September 30, 1976 and, in revised form, April 30, 1978.

AMS (MOS) subject classifications (1970). Primary 42A56; Secondary 44A25.

Key words and phrases. Mixed norm spaces, Littlewood-Paley function, Khinchine's inequality, Rademacher functions, Walsh-Fourier series, Hardy-Littlewood maximal function, lacunary maximal partial sum operator.

${ }^{1}$ Research was partially supported by NSF grant MPS 75-07586.

() 1979 American Mathematical Society 
Proof. For the first inequality, we consider the cases $p<r$ and $p>r$ separately. For $p<r$ it follows by Hölder's inequality and Khinchine's inequality for a single function that

$$
\begin{gathered}
\left(\int_{0}^{1}\left(\sum_{k=1}^{\infty}\left|f_{k}(x)\right|^{r}\right)^{p / r} d x\right)^{1 / p}<\left(\int_{0}^{1}\left(\sum_{k=1}^{\infty}\left|f_{k}(x)\right|^{r} d x\right)\right)^{1 / r} \\
=\left(\sum_{k=1}^{\infty} \int_{0}^{1}\left|f_{k}(x)\right|^{r} d x\right)^{1 / r}<C_{r}\left(\sum_{k=1}^{\infty}\left(\sum_{r=1}^{\infty}\left|a_{r}^{k}\right|^{2}\right)^{r / 2}\right)^{1 / r} .
\end{gathered}
$$

For $p>r$, it follows by Minkowski's inequality and Khinchine's inequality for a single function that

$$
\begin{aligned}
\left(\int_{0}^{1}\left(\sum_{k=1}^{\infty}\left|f_{k}(x)\right|^{r}\right)^{p / r} d x\right)^{1 / p} & <\left(\sum_{k=1}^{\infty}\left(\int_{0}^{1}\left|f_{k}(x)\right|^{p} d x\right)^{r / p}\right)^{1 / r} \\
& <C_{p}\left(\sum_{k=1}^{\infty}\left(\sum_{\nu=1}^{\infty}\left|a_{p}^{k}\right|^{2}\right)^{r / 2}\right)^{1 / r} .
\end{aligned}
$$

This proves the first inequality. To prove the second inequality, we consider the cases $p>r$ and $p<r$ separately. For $p>r$ it follows by Khinchine's inequality for a single function and Hölder's inequality that

$$
\begin{aligned}
& \left(\sum_{k=1}^{\infty}\left(\sum_{\nu=1}^{\infty}\left|a_{\nu}^{k}\right|^{2}\right)^{r / 2}\right)^{1 / r}<C_{r}\left(\sum_{k=1}^{\infty} \int_{0}^{1}\left|f_{k}(x)\right|^{r}\right)^{1 / r} \\
& \quad=C_{r}\left(\int_{0}^{1}\left(\sum_{k=1}^{\infty}\left|f_{k}(x)\right|^{r}\right)\right)^{1 / r}<C_{r}\left(\int_{0}^{1}\left(\sum_{k=1}^{\infty}\left|f_{k}(x)\right|^{r}\right)^{p / r}\right)^{1 / p} .
\end{aligned}
$$

Thus it remains to prove the second inequality only in the case $p<r$. To do this we use duality. Let $\left\{b_{r}^{k}\right\}$ denote an element of norm 1 in $l_{r}\left(l_{2}\right)$ where $1 / r+1 / r^{\prime}=1$. Then let $\left\{a_{r}^{k}\right\}_{v-1}^{N}, 1<k<K$, be any $N K$ numbers. Consider the corresponding $b_{k}^{r}$ and let $f_{1}, \ldots, f_{K}, g_{1}, \ldots, g_{K}$ be defined by $f_{k}=\sum_{v-1}^{N} a_{v}^{k_{v}} r_{v}(t)$ and $g_{k}=\sum_{v-1}^{N} b_{v}{ }^{k}, t(t)$. Then by Hölder's inequality with $(p, r)\left(p^{\prime}, r^{\prime}\right)$, and the first inequality of this lemma, we have

$$
\begin{aligned}
\left.K\left\{a_{v}^{k}\right\},\left\{b_{\nu}^{k}\right\}\right\rangle \mid & =\left|\int_{0}^{1} \sum_{k=1}^{K} f_{k}(x) g_{k}(x) d x\right| \\
& <\left(\int_{0}^{1}\left(\sum_{k=1}^{K} \mid f_{k}(x) r^{r}\right)^{p / r} d x\right)^{1 / p}\left(\int_{0}^{1}\left(\sum_{k=1}^{K}\left|g_{k}(x)\right|^{r}\right)^{p^{\prime} / r} d x\right)^{1 / r} \\
& <C_{p, r}\left(\int_{0}^{1}\left(\sum_{k=1}^{K}\left|f_{k}(x)\right|^{r}\right)^{p / r} d x\right)^{1 / p}\left(\sum_{k=1}^{K}\left(\sum_{\nu=1}^{N}\left|b_{v}^{k}\right|^{2}\right)^{r}\right)^{1 / r} \\
& <C_{p, r}\left(\int_{0}^{1}\left(\sum_{k=1}^{K}\left|f_{k}(x)\right|^{r}\right)^{p / r} d x\right)^{1 / p} .
\end{aligned}
$$


Taking the supremum over the unit ball of $l_{r}\left(l_{2}\right)$ we obtain

$$
\left(\sum_{k=1}^{K}\left(\sum_{\nu=1}^{N}\left|a_{\nu}^{k}\right|^{2}\right)^{r / 2}\right)^{1 / r} \leqslant C_{p, r}\left(\int_{0}^{1}\left(\sum_{k=1}^{K}\left|f_{k}(x)\right|^{r}\right)^{p / r} d x\right)^{1 / p} .
$$

Letting $N \rightarrow \infty$ and then $K \rightarrow \infty$ the lemma follows.

II. In this section we prove some basic estimates on certain multipliers. We note that partial results similar to these are proved in [11]. The proofs in [11] are for martingales and are based on a decomposition lemma of Gundy. We are able to give a short proof in view of recent work by $\mathbf{A}$. Cordoba and $\mathbf{C}$. Fefferman [2]. $S_{2}, f$ will denote the $2^{\nu}$ th Walsh series partial sum.

LEMMA 2. Let $a=\left\{a_{\nu}\right\}_{\nu=1}^{\infty}$ denote a sequence of real numbers with $\left\|\left\{a_{\nu}\right\}\right\|_{\infty}$ <1. Let $T_{a} f=\sum_{v=1}^{\infty} a_{\nu}\left(S_{2},-S_{2^{p-1}}\right) f$. Let $T_{a}\left\{f_{k}\right\}=\left\{T_{a} f_{k}\right\}_{k>1}$. Then for $1<$ $p, r<\infty$, there exists a constant $C_{p, r}$ such that

$$
\left(\int_{0}^{1}\left(\sum_{k=1}^{\infty}\left|T_{a} f_{k}(x)\right|^{r}\right)^{p / r} d x\right)^{1 / p} \leqslant C_{p, r}\left(\int_{0}^{1}\left(\sum_{k=1}^{\infty}\left|f_{k}(x)\right|^{r}\right)^{p / r} d x\right)^{1 / p} .
$$

In particular $C_{p, r}$ is independent of the sequence $a$.

PROoF. For a locally integrable function $f$, let $f^{\#}(x)=\sup _{x \in \omega}|\omega|^{-1} \int_{\omega} \mid f(t)$ $-f_{\omega}(t) \mid d t$ where $\omega$ is a dyadic interval and $f_{\omega}(t)=|\omega|^{-1} \int_{\omega} f(x) d x$ for $t \in \omega$. In view of results in [2], it suffices to prove that, for $1<p_{1}<\infty,\left(T_{a} f\right)^{\#}(x)$ $<C_{p_{1}}\left[\left(|f|^{p_{1}}\right)^{*}(x)\right]^{1 / p_{1}}$ with $C_{p_{1}}$ independent of $f$ and $a$. We note that $\left\|T_{a} f\right\|_{p_{1}}<$ $C_{p_{1}}\|f\|_{p_{1}}$ with $C_{p_{1}}$ independent of $f$ and $a$. This follows from the LittlewoodPaley theory for a single function. Let $\omega$ denote a dyadic interval and let $\omega^{*}$ denote the dyadic interval containing $\omega$ with $\left|\omega^{*}\right|=2|\omega|$. (If $\omega=[0,1]$, $\omega=\omega^{*}$.) Let $f=f_{1}+f_{2}$ where $f_{1}(\cdot)=f(\cdot) \chi_{\omega^{*}}(\cdot)$. Then for $x \in \omega$ we have

$$
\begin{aligned}
|\omega|^{-1} \int_{\omega}\left|T_{a} f_{1}(t)-\left(T_{a} f_{1}\right)_{\omega}(t)\right| d t & \\
& <2|\omega|^{-1} \int_{\omega}\left|T_{a} f_{1}(t)\right| d t<2\left(|\omega|^{-1} \int_{\omega}\left|T_{a} f_{1}(t)\right|^{p_{1}} d t\right)^{1 / p_{1}} \\
& <C_{p_{1}}\left(\left|\omega^{*}\right|^{-1} \int_{\omega^{*}}\left|f_{1}(t)\right|^{p_{1}} d t\right)^{1 / p_{1}}<C_{p_{1}}\left[\left(|f|^{p_{1}}\right)^{*}\right]^{1 / p_{1}}(x) .
\end{aligned}
$$

We recall from the theory of Walsh-Fourier series (see [5]) that $S_{2^{p+1}} f-S_{2^{2}} f$ $=f * \delta_{v}^{*}$ where $\delta_{v}^{*}$ has support in $\left[0,2^{-\nu}\right]$ and convolution is with respect to the dyadic structure on $[0,1]$. Now suppose $|\omega|=2^{-p}$. It then follows that, for $x \in \omega, S_{2^{r}} f_{2}(x)-S_{2^{p-1}} f_{2}(x)=0$ for $\nu>p$. Consequently, for $x \in \omega$, $T_{a} f_{2}(x)=\sum_{v=1}^{p-1} a_{v}\left(S_{2^{p}}-S_{2^{p-1}}\right)\left(f_{2}\right)(x)$. But $\sum_{v=1}^{p-1} a_{v}\left(S_{2^{p}}-S_{2^{p-1}}\right)\left(f_{2}\right)$ is a polynomial of degree at most $2^{\nu}$ and therefore is constant on intervals of length $2^{-\nu}$. It follows that 


$$
|\omega|^{-1} \int_{\omega}\left|T_{a} f_{2}(t)-\left(T_{a} f_{2}\right)_{\omega}(t)\right| d t=0
$$

and therefore

$$
\begin{aligned}
|\omega|^{-1} \int_{\omega}\left|T_{a} f(t)-\left(T_{a} f\right)_{\omega}(t)\right| d t & =|\omega|^{-1} \int_{\omega}\left|T_{a} f_{1}(t)-\left(T_{a} f_{1}\right)_{\omega}(t)\right| d t \\
& <C_{p_{1}}\left[\left(|f|^{p_{1}}\right)^{*}\right]^{1 / p_{1}}(x) .
\end{aligned}
$$

Taking the supremum norm over $\omega$ such that $x \in \omega$, we obtain $\left(T_{a} f\right)^{\#}(x)<$ $C_{p_{1}}\left[\left(|f|^{p_{1}}\right)^{*}\right]^{1 / p_{1}}(x)$. This completes the proof of Lemma 2.

We mention a special case of this result. We shall see later that more is actually true. Let $n$ be a fixed positive integer and let $S_{n} f(x)$ denote the $n$th partial sum of the Walsh-Fourier series of $f$. Then

$$
\left(\int_{0}^{1}\left(\sum_{k=1}^{\infty}\left|S_{n} f_{k}(x)\right|^{r}\right)^{p / r} d x\right)^{1 / p}<C_{p, r}\left(\int_{0}^{1}\left(\sum_{k=1}^{\infty}\left|f_{k}(x)\right|^{r}\right)^{p / r}\right)^{1 / p}
$$

where $C_{p, r}$ is independent of $n$ and $\left\{f_{k}\right\}$. This follows by writing $S_{n} f_{k}=$ $\omega_{n} S_{n}^{*}\left(f_{k} \omega_{n}\right)$ where $S_{n}^{*}$ denotes the modified partial sum (see [5]) and noting that $S_{n}^{*} f_{k}=T_{a} f_{k}$ where $a$ is a sequence of 0's and l's depending on $n$.

III. In this section we prove an inequality of Littlewood-Paley type for mixed norm spaces. For a function $f$ in $L^{p}(0 ; 1), p>1$, we define the Littlewood-Paley function $G f$ as

$$
G f(x)=\left(\sum_{\nu=1}^{\infty}\left|S_{2^{\prime} f} f(x)-S_{2^{p-1}} f(x)\right|^{2}\right)^{1 / 2}
$$

where $S_{2} f(x)=|\omega|^{-1} \int_{\omega} f(x) d x,|\omega|=2^{-\nu}, \omega \ni x$. Then for $f \in L^{p}(0 ; 1), 1<p$ $<\infty,\|G f\|_{p}<C_{p}\|f\|_{p}$ with $C_{p}$ independent of $f$. This is the Littlewood-Paley inequality for $L^{p}$ spaces [10].

THEOREM. Let $1<p, r<\infty$ and let $\left\{f_{k}\right\}$ be a sequence of integrable functions on $[0,1]$ with $\int_{0}^{1}\left(\sum_{k=1}^{\infty}\left|f_{k}(x)\right|^{r}\right)^{p / r} d x<\infty$. Then there exists a constant $C_{p, r}$ independent of $\left\{f_{k}\right\}$ such that

$$
\int_{0}^{1}\left(\sum_{k=1}^{\infty}\left|G f_{k}(x)\right|^{r}\right)^{p / r} d x<C_{p, r} \int_{0}^{1}\left(\sum_{k=1}^{\infty}\left|f_{k}(x)\right|^{r}\right)^{p / r} d x
$$

ProOF. This result is a simple consequence of the results in the previous two sections. For each $t \in[0,1]$ consider the sequence $\left\{\sum_{p=1}^{\infty} r_{p}(t)\left(S_{2} f_{k}(x)-\right.\right.$ $\left.\left.S_{2^{p-1}} f_{k}(x)\right)\right\}_{k}$. For each $t$ in $[0,1]$, Lemma 2 implies

$$
\begin{aligned}
& \int_{0}^{1}\left(\sum_{k=1}^{\infty} \mid \sum_{\nu=1}^{\infty} r_{p}(t)\right.\left.\left.\left(S_{2^{2}} f_{k}(x)-S_{2^{p-1}} f_{k}(x)\right)\right|^{r}\right)^{p / r} d x \\
&<C_{p, r} \int_{0}^{1}\left(\sum_{k=1}^{\infty}\left|f_{k}(x)\right|^{r}\right)^{p / r} d x
\end{aligned}
$$


Integrating with respect to $t$ and interchanging the order of integration, we obtain

$$
\begin{gathered}
\int_{0}^{1} \int_{0}^{1}\left(\sum_{k=1}^{\infty}\left|\sum_{\nu=1}^{\infty} r_{\nu}(t)\left(S_{2,} f_{k}(x)-S_{2^{p-1}} f_{k}(x)\right)\right|^{r}\right)^{p / r} d t d x \\
\leqslant C_{p, r} \int_{0}^{1}\left(\sum_{k=1}^{\infty}\left|f_{k}(x)\right|^{r}\right)^{p / r} d x .
\end{gathered}
$$

By Lemma 1 we have

$$
\begin{aligned}
\int_{0}^{1} \int_{0}^{1}\left(\sum_{k=1}^{\infty} \mid \sum_{\nu=1}^{\infty} r_{\nu}(t)\right. & \left.\left.\left(S_{2^{\prime}, f_{k}}(x)-S_{2^{p-1}} f_{k}(x)\right)\right|^{r}\right)^{p / r} d t d x \\
& >\tilde{C}_{p, r} \int_{0}^{1}\left(\sum_{k=1}^{\infty}\left(\sum_{\nu=1}^{\infty}\left|S_{2^{r}} f_{k}(x)-S_{2^{p-1}} f_{k}(x)\right|^{2}\right)^{r / 2}\right)^{p / r} d x \\
& =\tilde{C}_{p, r} \int_{0}^{1}\left(\sum_{k=1}^{\infty}\left|G f_{k}(x)\right|^{p / r} d x .\right.
\end{aligned}
$$

Hence

$$
\int_{0}^{1}\left(\sum_{k=1}^{\infty}\left|G f_{k}(x)\right|^{r}\right)^{p / r} d x<\tilde{\tilde{C}}_{p, r} \int_{0}^{1}\left(\sum_{k=1}^{\infty}\left|f_{k}(x)\right|^{r}\right)^{p / r} d x
$$

where $\tilde{\tilde{C}}_{p, r}$ is independent of $\left\{f_{k}\right\}$.

IV. For the basic properties of the Walsh-Paley system, we refer the reader to [5] or [7]. $S_{n} f(\cdot)$ will denote the $n$th partial sum of the Walsh-Paley-Fourier series of $f \in L^{1}[0,1]$.

In this section we extend a result of Paley to mixed norm spaces. Let $\left\{n_{\nu}\right\}_{\nu=1}^{\infty}$ denote a sequence of integers such that $n_{p+1}>q n_{\nu}, q>1, \nu=$ $1,2, \ldots$ For $f$ integrable on $[0,1]$, let $S f(x)=\sup _{\nu}\left|S_{n} f(x)\right|$. Paley [7] proved that for $1<p<\infty$, there exists a constant $A_{p, q}$ independent of $f$ such that $\|S f\|_{p}<A_{p q}\|f\|_{p}$. Our result is the following

THEOREM. Let $\left\{n_{p}\right\}_{\nu-1}^{\infty}$ denote a lacunary sequence as above. Let $\left\{f_{k}\right\}$ denote a sequence of integrable functions on $[0,1]$ such that $\int_{0}^{1}\left(\sum_{k=1}^{\infty}\left|f_{k}(x)\right|^{r}\right)^{p / r} d x<$ $\infty$. Let $1<p, r<\infty$. Then there exists a constant $A_{p, q, r}$ independent of $\left\{f_{k}\right\}$ such that

$$
\left(\int_{0}^{1}\left(\sum_{k=1}^{\infty}\left|S f_{k}(x)\right|^{r}\right)^{p / r} d x\right)^{1 / p}<A_{p, q, r}\left(\int_{0}^{1}\left(\sum_{k=1}^{\infty}\left|f_{k}(x)\right|^{r}\right)^{p / r}\right)^{1 / p} .
$$

REMARK. We note that in the special case $n_{p}=2^{y}$ our inequality reduces to dyadic analogue of the Fefferman-Stein inequality since $S_{2 f} f(x)$ is just the average of $f$ over the dyadic interval of length $2^{->}$which contains $x$. 
Proof. By splitting up the sequence $\left\{n_{p}\right\}_{p=1}^{\infty}$ into finitely many subsequences if necessary, we may assume $2^{y}<n_{p}<2^{y+1}$. The number of such subsequences depends only on $q$. For any $k, \nu>1$, let $d_{v}^{k}=S_{2^{p+1}} f_{k}-S_{2} f_{k}$. Then

$$
S_{n_{3}} f_{k}=S_{n_{3}}\left(f_{k}-S_{2,} f_{k}\right)+S_{2} f_{k} \equiv S_{n_{3}}\left(d_{v}^{k}\right)+S_{2 \cdot} f_{k}
$$

Then

$$
S f(x)<\sup _{\nu>1}\left|S_{n_{3}}\left(d_{\nu}^{k}\right)(x)\right|+\sup _{\nu>1}\left|S_{2 f_{k}}(x)\right|
$$

and therefore

$$
\begin{aligned}
\left(\sum_{k=1}^{\infty}\left|S f_{k}(x)\right|^{r}\right)^{1 / r}< & \left(\sum_{k=1}^{\infty}\left(\sup _{\nu>1}\left|S_{m_{3}}\left(d_{r}^{k}\right)(x)\right|\right)^{r}\right)^{1 / r} \\
& +\left(\sum_{k=1}^{\infty}\left(\sup _{\nu>1}\left|S_{22} f_{k}(x)\right|\right)^{r}\right)^{1 / r} .
\end{aligned}
$$

By the Fefferman-Stein inequality [3]

$$
\left(\int_{0}^{1}\left(\sum_{k=1}^{\infty}\left(\sup _{\nu>1}\left|S_{2,} f_{k}(x)\right|^{r}\right)^{p / r}\right)^{1 / p}<C_{p, r}\left(\int_{0}^{1}\left(\sum_{k=1}^{\infty}\left|f_{k}(x)\right|^{r}\right)^{p / r}\right)^{1 / p} .\right.
$$

Thus it suffices to prove

$$
\int_{0}^{1}\left(\sum_{k=1}^{\infty}\left(\sup _{\nu>1}\left|S_{n_{3}}\left(d_{p}^{k}\right)(x)\right|\right)^{r}\right)^{p / r}<C_{p, r} \int_{0}^{1}\left(\sum_{k=1}^{\infty}\left|f_{k}(x)\right|^{r}\right)^{p / r} d x
$$

We will first show that

$$
\begin{aligned}
\mid\{x \in[0,1]: & \left.\left(\sum_{k=1}^{\infty}\left(\sup _{\nu>1}\left|S_{n}\left(d_{\nu}^{k}\right)(x)\right|\right)^{r}\right)^{1 / r}>\lambda\right\} \mid \\
& <C_{r} \lambda^{-p} \int_{0}^{1}\left(\sum_{k=1}^{\infty}\left|G f_{k}(x)\right|^{r}\right)^{p / r} d x .
\end{aligned}
$$

Combining this with the result in \$III gives

$$
\begin{gathered}
\left|\left\{x \in[0,1]:\left(\sum_{k=1}^{\infty}\left(\sup _{\nu>1}\left|S_{n}\left(d_{\nu}^{k}\right)(x)\right|\right)^{r}\right)^{1 / r}>\lambda\right\}\right| \\
<\tilde{C}_{p, r} \lambda^{-p} \int\left(\sum_{k=1}^{\infty}\left|f_{k}(x)\right|^{r}\right)^{p / r} d x .
\end{gathered}
$$

An interpolation argument similar to the Marcinkiewicz theorem yields (*). By the Calderón-Zygmund lemma there exists a sequence $\left\{\omega_{j}\right\}$ of mutually disjoint dyadic intervals of $[0,1]$ which satisfy

(i) $\lambda<\left(\left|\omega_{j}\right|^{-1} \int_{\omega_{j}}\left(\sum_{k=1}^{\infty} G f_{k}(x)^{r}\right)^{p / r} d x\right)^{1 / p}<2 \lambda$,

(ii) $\sum_{j=1}^{\infty}\left|\omega_{j}\right|<\lambda^{-p} \int_{0}^{1}\left(\sum_{k=1}^{\infty} G f_{k}(x)^{r}\right)^{p / r} d x$, 
LITTLEWOOD-PALEY THEORY FOR MIXED NORM SPACES

119

(iii) $\left(\sum_{k=1}^{\infty} G f_{k}(x)^{r}\right)^{1 / r} \leqslant \lambda$ for a.e. $x \notin \cup_{j} \omega_{j}$.

Fix a large positive integer $M$. We will prove the stated inequality with $\sup _{1<v<M}\left|S_{n_{3}}\left(d_{v}^{k}\right)(x)\right|$ in place of $\sup _{\nu>1}\left|S_{n_{3}}\left(d_{v}^{k}\right)(x)\right|$, and $C_{r}$ will be independent of $M$. The desired inequality will then follow by letting $M$ tend to $\infty$. Let $\Omega=\cup_{j} \omega_{j}$. We define, for $1<\nu<M$,

$$
g_{v}^{k}(x)= \begin{cases}\omega_{n_{p}}(x)\left(\left|\omega_{j}\right|^{-1} \int_{\omega_{j}} \omega_{n_{3}}(t) d_{p}^{k}(t) d t\right) & \text { if } x \in \omega_{j}, \\ d_{\nu}^{k}(x) & \text { if } x \notin \Omega .\end{cases}
$$

Let $b_{\nu}^{k}=d_{v}^{k}-g_{v}^{k}, k>1,1<\nu<M$. As in [8] we note that for each $k>1$ and $x \notin \Omega, S_{n_{2}}\left(d_{\nu}^{k}\right)(x)=S_{n_{2}}\left(g_{\nu}^{k}\right)(x), 1<\nu<M$. Thus

$$
\begin{aligned}
& \left|\left\{x \in[0,1]:\left(\sum_{k=1}^{\infty}\left(\sup _{1<\nu<M}\left|S_{m_{2}}\left(d_{\nu}^{k}\right)(x)\right|\right)^{r}\right)^{1 / r}>\lambda\right\}\right| \\
& \leq|\Omega|+\left|\left\{x \in[0,1] \backslash \Omega:\left(\sum_{k=1}^{\infty}\left(\sup _{1<\nu<M}\left|S_{n_{3}}\left(g_{r}^{k}\right)(x)\right|^{r}\right)\right)^{1 / r}>\lambda\right\}\right| \\
& <|\Omega|+\left|\left\{x \in[0,1] \backslash \Omega:\left(\sum_{k=1}^{\infty}\left(\sum_{\nu=1}^{M}\left|S_{n_{3}}\left(g_{\nu}^{k}\right)(x)\right|^{2}\right)^{r / 2}\right)^{1 / r}>\lambda\right\}\right| \\
& <|\Omega|+\lambda^{-r} \int_{[0,1] \backslash \Omega}\left(\sum_{k=1}^{\infty}\left|S_{n_{3}}\left(g_{\nu}^{k}\right)(x)\right|^{2}\right)^{r / 2} d x \\
& <|\Omega|+\lambda^{-r} \int_{0}^{1} \sum_{k=1}^{\infty}\left(\sum_{\nu=0}^{M}\left|S_{n_{\nu}} g_{\nu}^{k}(x)\right|^{2}\right)^{r / 2} d x \\
& =|\Omega|+\lambda^{-r} \sum_{k=1}^{\infty} \int_{0}^{1}\left(\sum_{\nu=0}^{M}\left|S_{n} g_{\nu}^{k}(x)\right|^{2}\right)^{r / 2} d x .
\end{aligned}
$$

We first note that $S_{2^{\prime}} g_{\nu}^{k}(x) \equiv 0$, i.e., $\int_{\omega} g_{\nu}^{k}(x) d x=0$ for every dyadic interval $\omega$ with $|\omega|=2^{-k}$. If $\omega \cap \Omega=\varnothing$, this is immediate since $\int_{\omega} g_{\nu}^{k}(x) d x=$ $\int_{\omega} d_{\nu}^{k}(x) d x=0$ since each term in $d_{\nu}^{k}$ splits on $\omega$. If $\omega=\omega_{j} \in \Omega$ for some $j$, $\int_{\omega} g_{\nu}^{k}(x) d x=C \int_{\omega} \omega_{n_{2}}(x) d x=0$ ( $C$ is a constant). Finally suppose $\omega \cap \Omega \neq$ $\varnothing$ but $\omega \neq \omega_{j}$ for any $\omega_{j} \in \Omega$. Let $\omega \cap \Omega=\cup_{j^{\prime}} \omega_{j^{\prime}}$. Note that $\left|\omega_{j^{\prime}}\right|<2^{->-1}$ for each $j^{\prime}$. Thus

$$
\begin{aligned}
\int_{\omega} g_{\nu}^{k}(x) d x & =\sum_{j^{\prime}} \int_{\omega_{j^{\prime}}} g_{\nu}^{k}(x) d x+\int_{\omega \backslash \cup \omega_{j}} d_{\nu}^{k}(x) d x \\
& =\sum_{j^{\prime}} \int_{\omega_{j}}\left(\frac{1}{\left|\omega_{j^{\prime}}\right|} \omega_{n^{\prime}}(x) \int_{\omega_{j}} \omega_{n^{\prime}}(t) d_{\nu}^{k}(t) d t\right) d x+\int_{\omega \backslash \cup \omega_{j}} d_{\nu}^{k}(x) d x \\
& =\sum_{j^{\prime}} \int_{\omega_{j}} \frac{1}{\left|\omega_{j^{\prime}}\right|} \int_{\omega_{j}} d_{\nu}^{k}(t) d t d x+\int_{\omega \backslash \cup, \omega_{j}} d_{\nu}^{k}(x) d x
\end{aligned}
$$


(since $\omega_{n_{3}}(\cdot)$ is constant on each $\omega_{j}$ )

$$
=\sum_{j^{\prime}} \int_{\omega_{j}} d_{\nu}^{k}(t) d t+\int_{\omega \backslash \cup j^{\prime} \omega_{j}} d_{\nu}^{k}(x) d x=\int_{\omega} d_{\nu}^{k}(t) d t=0 .
$$

Thus $S_{n} g_{\nu}^{k}(x)=S_{n} g_{\nu}^{k}(x)-S_{2}, g_{\nu}^{k}(x)$. By a theorem of R. E. A. C. Paley on partial blocks [7, Lemma 13] there exists a constant $C_{r}$ depending only on $r$ such that

$$
\int_{0}^{1}\left(\sum_{\nu=0}^{M}\left|S_{n_{2}} g_{\nu}^{k}(x)\right|^{2}\right)^{r / 2} d x<C_{r} \int_{0}^{1}\left(\sum_{\nu=0}^{M}\left|S_{2^{p+1}} g_{\nu}^{k}(x)\right|^{2}\right)^{r / 2} d x
$$

Returning to our inequalities, we now have

$$
\begin{aligned}
\mid\left\{x:\left(\sum_{k=1}^{\infty}\right.\right. & \left.\left.\left(\sup _{1<\nu<M}\left|S_{n}\left(d_{\nu}^{k}\right)(x)\right|\right)^{r}\right)>\lambda\right\} \mid \\
& <|\Omega|+C_{r} \lambda^{-r} \int_{0}^{1} \sum_{k=1}^{\infty}\left(\sum_{\nu=0}^{M}\left|S_{2^{p+1}} g_{\nu}^{k}(x)\right|^{2}\right)^{r / 2} d x .
\end{aligned}
$$

We now estimate $\sum_{k=1}^{\infty}\left(\sum_{\nu=1}^{M}\left|S_{2^{p+1}} g_{\nu}^{k}(x)\right|^{2}\right)^{r / 2}$ separately for $x \notin \Omega$ and $x \in \Omega$. We first consider the case $x \notin \Omega$. Let $\omega$ denote the dyadic interval of length $2^{-\nu-1}$ which contains $x$. Then $\omega \notin\left\{\omega_{j}\right\}$ and the argument above shows that $\int_{\omega} g_{\nu}^{k}(x) d x=\int_{\omega} d_{\nu}^{k}(x) d x$. Thus

$$
\begin{aligned}
\sum_{k=1}^{\infty}\left(\sum_{\nu=0}^{M}\left|S_{2^{p+1}} g_{\nu}^{k}(x)\right|^{2}\right)^{r / 2} & =\sum_{k=1}^{\infty}\left(\sum_{\nu=0}^{M}\left(\frac{1}{|\omega|}\left|\int_{\omega} g_{\nu}^{k}(x) d x\right|\right)^{2}\right)^{r / 2} \\
& <\sum_{k=1}^{\infty}\left(\sum_{\nu=0}^{M}\left(\frac{1}{|\omega|} \int_{\omega}\left|d_{\nu}^{k}(x)\right|\right)^{2}\right)^{r / 2} \\
& =\sum_{k=1}^{\infty}\left(\sum_{\nu=0}^{M}\left|d_{\nu}^{k}(x)\right|^{2}\right)^{r / 2}
\end{aligned}
$$

(since $d_{r}^{k}(x)$ is constant on dyadic intervals of length $2^{->-1}$ )

$$
<\sum_{k=1}^{\infty}\left(\sum_{\nu=0}^{\infty}\left|d_{\nu}^{k}(x)\right|^{2}\right)^{r / 2}=\sum_{k=1}^{\infty} G f_{k}(x)^{r}
$$

By property (iii) of the decomposition, it follows that for a.e. $x \notin \Omega$ we have 


$$
\begin{aligned}
\sum_{k=1}^{\infty}\left(\sum_{r=0}^{M}\left|S_{2^{r+1}} g_{v}^{k}(x)\right|^{2}\right)^{r / 2} & <\sum_{k=1}^{\infty} G f_{k}(x)^{r} \\
& <\left(\sum_{k=1}^{\infty} G f_{k}(x)^{r}\right)^{p / r}\left(\sum_{k=1}^{\infty} G f_{k}(x)^{r}\right)^{(r-p) / r} \\
& <\lambda^{r-p}\left(\sum_{k=1}^{\infty} G f_{k}(x)^{r}\right)^{p / r}
\end{aligned}
$$

We now consider the case $x \in \Omega$. Let $\bar{\omega}$ denote the element of $\Omega$ which contains $x$ and let $|\bar{\omega}|=2^{-s}$. Then letting $\omega_{\nu}$ denote the dyadic interval of length $2^{-v}$ containing $x$, we have

$$
\begin{aligned}
\left(\sum_{\nu=0}^{S-2}\left|S_{2^{p+1}} g_{\nu}^{k}(x)\right|^{2}\right)^{r / 2} & =\left(\sum_{\nu=0}^{S-2}\left(\frac{1}{\left|\omega_{\nu+1}\right|}\left|\int_{\omega_{\nu+1}} g_{\nu}^{k}(x) d x\right|\right)^{2}\right)^{r / 2} \\
& =\left(\sum_{\nu=0}^{S-2}\left(\frac{1}{\left|\omega_{\nu+1}\right|}\left|\int_{\omega_{\nu+1}} d_{\nu}^{k}(x) d x\right|\right)^{2}\right)^{r / 2}
\end{aligned}
$$

(since each $\omega_{p+1} \neq \omega_{j}$ and the earlier argument applies)

$$
<\left(\sum_{\nu=0}^{S-2}\left|d_{\nu}^{k}(x)\right|^{2}\right)^{r / 2}=\left(\frac{1}{|\bar{\omega}|} \int_{\bar{\omega}}\left(\sum_{\nu=0}^{S-2}\left|d_{\nu}^{k}(x)\right|^{2}\right)^{1 / 2} d x\right)^{r}
$$

(since each $d_{\nu}^{k}(\cdot)$ is constant on $\bar{\omega}$ ). Also we have

$$
\begin{aligned}
& \left(\sum_{v=S-1}^{M}\left|S_{2^{p+1}} g_{v}^{k}(x)\right|^{2}\right)^{r / 2}=\left(\sum_{\nu=S-1}^{M} \frac{1}{\left|\omega_{v+1}\right|}\left|\int_{\omega_{p+1}} g_{v}^{k}(x) d x\right|^{2}\right)^{r / 2} \\
& =\left(\sum_{\nu=S-1}^{M} \frac{1}{\left|\omega_{\nu+1}\right|}\left|\int_{\omega_{\nu+1}} \omega_{n_{3}}(x) \frac{1}{|\bar{\omega}|} \int_{\bar{\omega}} \omega_{n_{3}}(t) d_{\nu}^{k}(t) d t d x\right|^{2}\right)^{r / 2} \\
& <\left(\sum_{\nu=S-1}^{M}\left(\frac{1}{\left|\omega_{\nu+1}\right|} \int_{\omega_{\nu+1}} \frac{1}{|\bar{\omega}|}\left|\int_{\bar{\omega}} \omega_{n_{3}}(t) d_{\nu}^{k}(t) d t\right| d x\right)^{2}\right)^{r / 2} \\
& =\left(\sum_{\nu=S-1}^{M}\left(\frac{1}{|\bar{\omega}|}\left|\int_{\bar{\omega}} \omega_{n_{3}}(t) d_{\nu}^{k}(t) d t\right|\right)^{2}\right)^{r / 2} \\
& <\left(\sum_{r=S-1}^{M}\left(\frac{1}{|\bar{\omega}|} \int_{\bar{\omega}}\left|d_{\nu}^{k}(t)\right| d t\right)^{2}\right)^{r / 2} \\
& <\left(\frac{1}{|\bar{\omega}|}\left(\int_{\bar{\omega}} \sum_{\nu=S-1}^{M}\left|d_{\nu}^{k}(t)\right|^{2}\right)^{1 / 2}\right)^{r} \text {. }
\end{aligned}
$$


Returning to our inequality we have for $x \in \Omega$, say $x \in \bar{\omega}$,

$$
\begin{aligned}
& \sum_{k=1}^{\infty}\left(\sum_{\nu=0}^{M}\left|S_{2^{p+1}} g_{\nu}^{k}(x)\right|^{2}\right)^{r / 2} \\
& <\left(\sum_{k=1}^{\infty}\left(\sum_{\nu=0}^{S-2}\left|S_{2^{p+1}} g_{\nu}^{k}(x)\right|^{2}\right)^{1 / 2}+\left(\sum_{\nu=S-1}^{M}\left|S_{2^{p+1}} g_{\nu}^{k}(x)\right|^{2}\right)^{1 / 2}\right)^{r} \\
& <2^{r}\left\{\sum_{k=1}^{\infty}\left(\sum_{\nu=0}^{S-2}\left|S_{2^{p+1}} g_{\nu}^{k}(x)\right|\right)^{r / 2}+\sum_{k=1}^{\infty}\left(\sum_{\nu=S-1}^{M}\left|S_{2^{p+1}} g_{\nu}^{k}(x)\right|^{2}\right)^{r / 2}\right\} \\
& <2^{r}\left\{\sum_{k=1}^{\infty}\left(\frac{1}{|\bar{\omega}|} \int_{\bar{\omega}}\left(\sum_{\nu=0}^{S-2}\left|d_{\nu}^{k}(x)\right|^{2}\right)^{1 / 2} d x\right)^{r}\right. \\
& \left.+\sum_{k=1}^{\infty}\left(\frac{1}{|\bar{\omega}|} \int_{\bar{\omega}}\left(\sum_{\nu=S-1}^{M}\left|d_{\nu}^{k}(x)\right|^{2}\right)^{1 / 2} d x\right)\right\} \\
& <2\left\{\left[\frac{1}{|\bar{\omega}|} \int_{\bar{\omega}}\left(\sum_{k=1}^{\infty}\left(\sum_{\nu=0}^{s-2}\left|d_{\nu}^{k}(x)\right|^{2}\right)^{r / 2}\right)^{1 / r}\right]^{r}\right. \\
& \left.+\left[\frac{1}{|\bar{\omega}|} \int_{\bar{\omega}}\left(\sum_{k=1}^{\infty}\left(\sum_{\nu=-1}^{M}\left|d_{\nu}^{k}(x)\right|^{2}\right)^{r / 2}\right)^{1 / r}\right]\right\} \\
& <2^{r}\left\{\left[\frac{1}{|\bar{\omega}|} \int_{\bar{\omega}}\left(\sum_{k=1}^{\infty} G f_{k}^{r}\right)^{1 / r}\right]^{r}+\left[\frac{1}{|\bar{\omega}|} \int_{\bar{\omega}}\left(\sum_{k=1}^{\infty} G f_{k}^{r}\right)^{1 / r}\right]^{r}\right\} \\
& <2^{r+1}[2 \lambda]^{r}=2^{2 r+1} \lambda^{r}
\end{aligned}
$$

by property (i) of the decomposition. This is the estimate for $x \notin \Omega$. Combining these results with inequality $(\dagger)$ we have

$$
\begin{aligned}
& \left|\left\{x:\left(\sum_{k=1}^{\infty}\left(\sup _{1<\nu<M}\left|S_{n_{3}}\left(d_{\nu}^{k}\right)(x)\right|\right)^{r}\right)^{1 / r}>\lambda\right\}\right| \\
& <|\Omega|+C_{r} \lambda^{-r} \int_{0}^{1} \sum_{k=1}^{\infty}\left(\sum_{\nu=0}^{M}\left|S_{2^{p+1}} g_{\nu}^{k}(x)\right|^{2}\right)^{r / 2} d x \\
& =|\Omega|+C_{p} \lambda^{-r} \int_{\Omega} \sum_{k=1}^{\infty}\left(\sum_{\nu=0}^{M}\left|S_{2^{p+1}} g_{\nu}^{k}(x)\right|^{2}\right)^{r / 2} d x \\
& \quad+C_{r} \lambda^{-r} \int_{[0,1] \Omega} \sum_{k=1}^{\infty}\left(\sum_{\nu=0}^{M}\left|S_{2^{p+1}} g_{\nu}^{k}(x)\right|^{2}\right)^{r / 2} d x
\end{aligned}
$$




$$
\begin{aligned}
& <|\Omega|\left\{1+C_{r} \lambda^{-r}\left(2^{2 r+1} \lambda^{r}\right)\right\}+C_{r} \lambda^{-r} \int_{[0,1] \backslash \Omega} \sum_{k=1}^{\infty} G f_{k}(x)^{r} d x \\
& <|\Omega|\left\{1+C_{r} 2^{2 r+1}\right\}+C_{r} \lambda^{-r} \lambda^{(r-p)} \int_{[0,1] \wedge \Omega}\left(\sum_{k=1}^{\infty} G f_{k}(x)^{r}\right)^{p / r} d x \\
& <|\Omega|\left\{1+C_{r} 2^{2 r+1}\right\}+C_{r} \lambda^{-p} \int_{0}^{1}\left(\sum_{k=1}^{\infty} G f_{k}(x)^{r}\right)^{p / r} d x \\
& <\lambda^{-p}\left\{1+C_{r} 2^{2 r+1}+C_{r}\right\} \int_{0}^{1}\left(\sum_{k=1}^{\infty} G f_{k}(x)^{r}\right)^{p / r} d x
\end{aligned}
$$

by property (ii) of the decomposition. Letting $M$ tend to $\infty$, we obtain

$$
\begin{gathered}
\left|\left\{x \in[0,1]:\left(\sum_{k=1}^{\infty}\left(\sup _{\nu}\left|S_{n_{3}}\left(d_{\nu}^{k}\right)(x)\right|\right)^{r}\right)^{1 / r}>\lambda\right\}\right| \\
<\tilde{C}_{r} \lambda^{-p} \int_{0}^{1}\left(\sum_{k=1}^{\infty} G f_{k}(x)^{r}\right)^{p / r} d x
\end{gathered}
$$

which is the desired inequality.

REMARKS. (i) In the case $f_{k}=0$ for $k>2$, our proof reduces to a direct proof of the strong type $(p, p)$ inequality for the lacunary maximal partial sum operator for Walsh-Fourier series. The only proof in the literature known to the author is that of R. E. A. C. Paley which is not based on the Calderón-Zygmund theory.

(ii) The above proof can be easily modified to yield a partial analogue of the result in [8]:

$$
\begin{gathered}
\left|\left\{x \in[0,1]:\left(\sum_{k=1}^{\infty}\left(\sup _{k}\left|S_{n_{k}}\left(d_{k}^{\nu}\right)(x)\right|\right)^{r}\right)^{1 / r}>\lambda\right\}\right| \\
<C_{r} \lambda^{-1} \int_{0}^{1}\left(\sum_{k=1}^{\infty} G f_{k}(x)^{r}\right)^{1 / r} d x
\end{gathered}
$$

Whether the full analogue is true depends upon integrability conditions between $\left(\sum_{k=1}^{\infty} H f_{k}(x)^{r}\right)^{1 / r}$ and $\left(\sum_{k=1}^{\infty} G f_{k}(x)^{r}\right)^{1 / r}$ where $H$ denotes the Hardy-Littlewood maximal function. Such results are unknown to the author.

(iii) A natural question which arises from this work is whether analogous inequalities can be proved for the full maximal partial sum operator.

ADDENDUM. A positive answer to the question raised in (iii) was recently announced by Jose L. Rubio de Francia, Universidad de Zaragoza (Spain) in the Notices Amer. Math. Soc. 26 (1979), p. A-216. 


\section{REFERENCES}

1. A. Benedeck and R. Panzone, The spaces $L^{p}$ with mixed norm, Duke Math. J. 29 (1961), 301-324.

2. A. Cordoba and C. Fefferman, A weighted norm inequality for singular integrals, Studia Math. 57 (1976), 97-101.

3. C. Fefferman and E. M. Stein, Some maximal inequalities, Amer. J. Math. 93 (1971), 107-115.

4. R. John, Weighted norm inequalities for singular and hypersingular integrals, Doctoral Dissertation, Rutgers University, New Brunswick, N. J., 1975.

5. R. A. Hunt, Almost everywhere convergence of Walsh-Fourier series of $L^{2}$ functions, Proc. Internat. Congress Math. (Nice, 1970), vol. 2, 1971, pp. 655-661.

6. R. A. Hunt and W. S. Young, A weighted norm inequality for Fourier series, Bull. Amer. Math. Soc. 80 (1974), 274-277.

7. R. E. A. C. Paley, A remarkable series of orthogonal functions. I, Proc. London Math. Soc. 34 (1932), 241-264.

8. D. Pankratz, Almost evenywere conoergence of lacunary partial sums of Walsh-Fourier series, Doctoral Dissertation, Purdue University, Lafayette, Ind., 1974.

9. E. M. Stein, Singular integrals and differentiability properties of functions, Princeton Univ. Press, Princeton, N. J., 1970.

10. - Topics in harmonic analysis related to the Littlewood-Paley theory, Ann. of Math. Studies, no. 63, Princeton Univ. Press, Princeton, N. J., 1970.

11. W. S. Young, Some maximal inequalities for martingales, part of Doctoral Dissertation, Purdue University, Lafayette, Ind., 1973.

12. A. Zygmund, Trigonometric series, Vols. I, II, 2nd ed., Cambridge Univ. Press, London and New York, 1959.

Department of Mathematics, Universtty of Grorgi, Athens, Georgu 30602 